\title{
Low frequency stall modes of a radial vaned diffuser flow
}

\author{
Victor Moënne-Loccoz ${ }^{1}$, Isabelle Trébinjac ${ }^{1}$, Nicolas Poujol ${ }^{2, *}$, and Pierre Duquesne ${ }^{1}$ \\ ${ }^{1}$ Laboratoire de Mécanique des Fluides et d'Acoustique, Ecole Centrale de Lyon, 69134 Ecully, France \\ 2 Safran Helicopter Engines, 64510 Bordes, France
}

Received: 15 October 2019 / Accepted: 24 March 2020

\begin{abstract}
The present paper aims at providing an experimental analysis of the path to surge of a centrifugal compressor stage designed and built by Safran Helicopter Engines. Depending on the rotation speed of the compressor, two distinct flow patterns are observed in the radial diffuser at stabilized operating points near the surge, an asymmetric and a symmetric pattern. At medium rotation speed, the alternate pattern consisting of a two-channel pattern in the radial diffuser develops. One passage over two is stalled, the adjacent passage is free and this pattern replicates over the whole circumference while pulsing at a frequency of roughly $12 \mathrm{~Hz}$ which is close to the Helmholtz frequency of the test rig. By lowering the rotation speed, the two-channel pattern fades away and gives way to a periodical behavior of the radial diffuser passages called symmetric mode. The flow in each channel is identical presenting a stalled behavior pulsating in phase at a higher frequency of roughly $42 \mathrm{~Hz}$. The two $12 \mathrm{~Hz}$ and $42 \mathrm{~Hz}$ modes are described and their existences are imputed to a lock-in of the natural frequencies of the instabilities with the acoustic modes of the test rig.
\end{abstract}

Keywords: stall / centrifugal compressor / diffuser / experimental

\section{Introduction}

Compressor operating range is limited at low mass flow by severe aerodynamic instabilities which occur as the flow inertia becomes too weak to counter the adverse pressure gradient. The most feared instability is called surge. It consists of a global reversal of the flow which may result in the destruction of the entire engine.

In axial compressors, as the mass flow is reduced, a typical path to surge involves rotating stall which then triggers surge. Centrifugal compressors show much more complex path to surge as stall of one of the subcomponent does not systematically trigger the instability of the whole compressor [1]. Although general trends may have been established from previous studies, they are flawed in many cases where centrifugal compressors show peculiar behaviors near their stability limits especially at partial rotation speeds [2-4]. Unfortunately, most of the time, no clear explanation on the mechanism of surge onset is found and many questions remain about the cause of these complex paths to surge.

Several authors reconsider the impact of the test rig geometry on surge. The link between the surge characteristics (typically frequency and amplitude) and the geometry of the test bench no longer needs to be proven. Greitzer

\footnotetext{
* e-mail: nicolas.poujol@ec-lyon.fr
}

$[5,6]$ theoretically and experimentally showed this dependency through the famous parameter B, which was confirmed in numerous studies [7-9]. The current questions reside on the onset of the precursory instabilities and their possible evolution into surge. On several occasions, the acoustics specific modes of the test rig seem to contribute to the instability of the compressor. A lock-in between the frequency of the instabilities and those modes may occur and trigger the surge of the compressor through resonance. Ziada et al. [10] showed this type of acoustic lock-in between the vortex shedding from the struts with the acoustic modes of the inlet volute in a $35 \mathrm{MW}$ turbocharger composed of 7 stages. This resulted in large pressure fluctuations of a frequency around $135 \mathrm{~Hz}$ close to the surge line of the compressor. Another example is given by Brandstetter et al. [11] on a UHBR-fan application. In this case, the acoustics modes of the exhaust system force a lock-in of aerodynamic effects in the rotor due to mistuning of the rotor blades. This lock-in amplifies the alternation provoked by the mistuning and finally triggers critical instabilities.

The compressor studied in this paper shows different behaviors involving different frequencies depending on the rotation speed, as the compressor approaches its stability limit. The present paper aims to present, analyze and interpret them. To do this, the compressor and the associated measurements are first presented. Then the path to surge at two selected rotation speeds is described. 


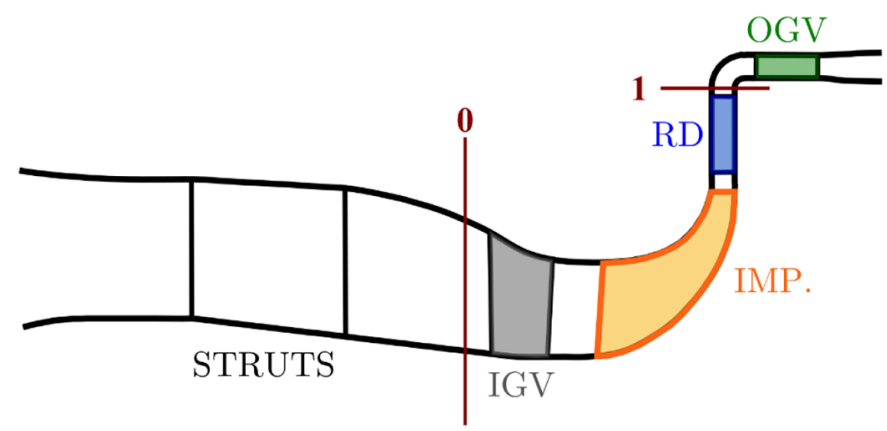

Fig. 1. Meridional view of the compressor.

The analysis of the involved low frequency modes is performed and the contribution of an acoustic coupling is discussed.

\section{Experimental setup}

\subsection{Test case}

The test case is a centrifugal compressor stage designed and built by SAFRAN Helicopter Engines within the framework of a research program. The compressor is composed of inlet guide vanes (IGV), a backswept splittered unshrouded impeller (IMP), a splittered vaned radial diffuser (RD) with an even number of vanes and axial outlet guide vanes (OGV). A meridional sketch of the stage is given in Figure 1.

The overall stage total to static pressure ratio is around 4. The Mach number based on the peripheral velocity at the exit radius of the impeller is around 1.2 at maximal rotation speed.

\subsection{Measurements and uncertainties}

The compressor stage is mounted on a $1 \mathrm{MW}$ test rig at the Laboratoire de Mécanique des Fluides et d'Acoustique, École Centrale de Lyon, France. 130 steady sensors (temperature, pressure) are used for the monitoring and the overall performance measuring. The rotation speed, the flow rate, the pressure and the temperature are measured at $\pm 0.01 \%, \pm 0.5 \%, \pm 0.05 \%$ and $\pm 0.5 \mathrm{~K}$ respectively. The uncertainties of the combined quantities are then evaluated using propagation formulas.

In order to capture the unsteady fluctuations of the flow, 52 unsteady pressure sensors manufactured by Kulite Semiconductor Product, Inc. are installed at different locations both on the shroud and the hub of the compressor. The protection grids were removed to maximize the natural frequency of the probes allowing pressure measurements up to $150 \mathrm{kHz}$ with an acquisition frequency of $500 \mathrm{kHz}$. Concerning the unsteady pressure measurements, the average value of the uncertainties of the fluctuations are evaluated at less than $0.7 \%$ of the peak-to-peak pressure fluctuation of the phase averaged signal.

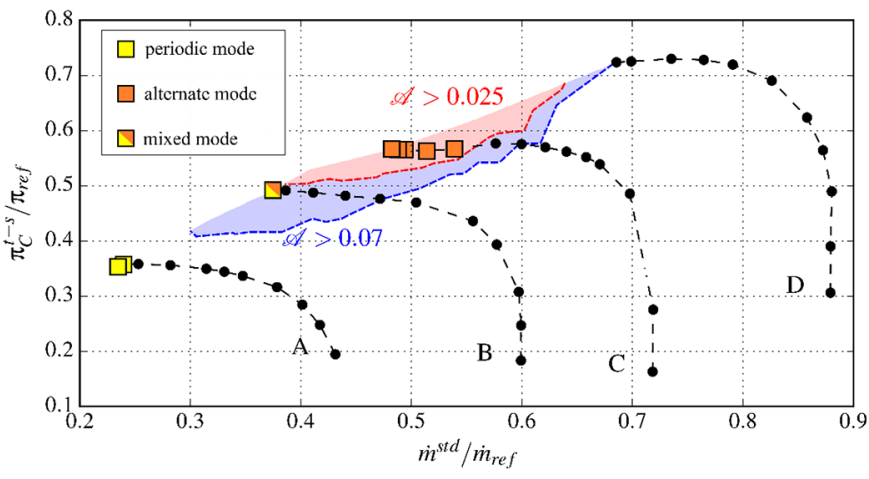

Fig. 2. Diffuser flow modes at partial rotation speeds.

\section{Path to surge}

The analysis proposed in this paper focuses on the operation of the compressor at partial rotation speeds (50-80\% of nominal speed). Figure 2 shows the performance map of the compressor at four iso-speeds. It depicts the total-to-static pressure ratio defined in equation (1) as a function of the corrected mass flow defined in equation (2). Both quantities are reduced by reference values.

$$
\begin{gathered}
\pi_{c}^{t-s}=\frac{P_{1}}{P_{t 0}} \\
\dot{m}^{s t d}=\dot{m} \sqrt{\frac{T_{t 0}}{T_{t}^{s t d}}} \frac{P_{t}^{s t d}}{P_{t 0}} .
\end{gathered}
$$

This performance map is also divided into 3 areas colored in white, blue and pink in Figure 2. Indeed, previous work [12-14] on the compressor has shown the possible setting of an alternate stall pattern over two adjacent channels in the radial diffuser at partial rotation speed. In such an alternate pattern, one diffuser passage is stalled, the adjacent passage is free and this pattern is repeated all around the circumference. Based on the circumferential static (steady and unsteady) pressure distribution, an indicator - the alternate rate $\mathcal{A}$ - has been developed to determine the establishment and magnitude of the alternate pattern. More information on the calculation of the alternate rate $\mathcal{A}$ and the thresholds used below is given in [15] ( $\mathcal{A}$ is named asymmetry rate instead of alternate rate in [15]). The superimposition of this indicator on the performance map, shows a very localized area of the setting of the alternate pattern. Isospeeds $\mathrm{A}$ and $\mathrm{D}$ show no sign of alternation (white area ; $\mathcal{A}<0.007)$ on the whole characteristic while isospeeds $\mathrm{B}$ and $\mathrm{C}$ show respectively moderate (blue area $; 0.007<\mathcal{A}$ $<0.025$ ), and high level (pink area ; $\mathcal{A}>0.025$ ) of alternation.

Depending on the value of this alternate rate, distinct low frequency flow modes are registered in the diffuser near surge: 


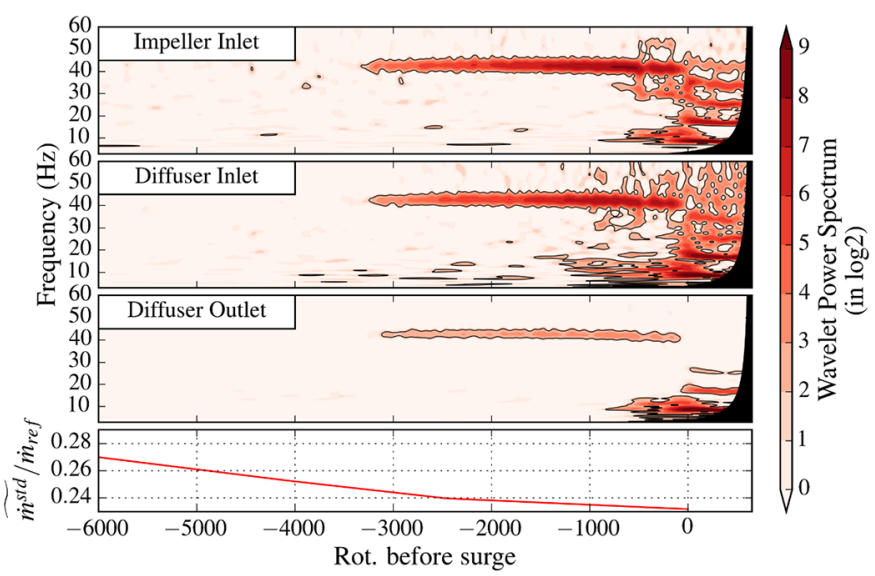

Fig. 3. Wavelet Power Spectrum during throttling ramp from stable condition to surge at isospeed A.

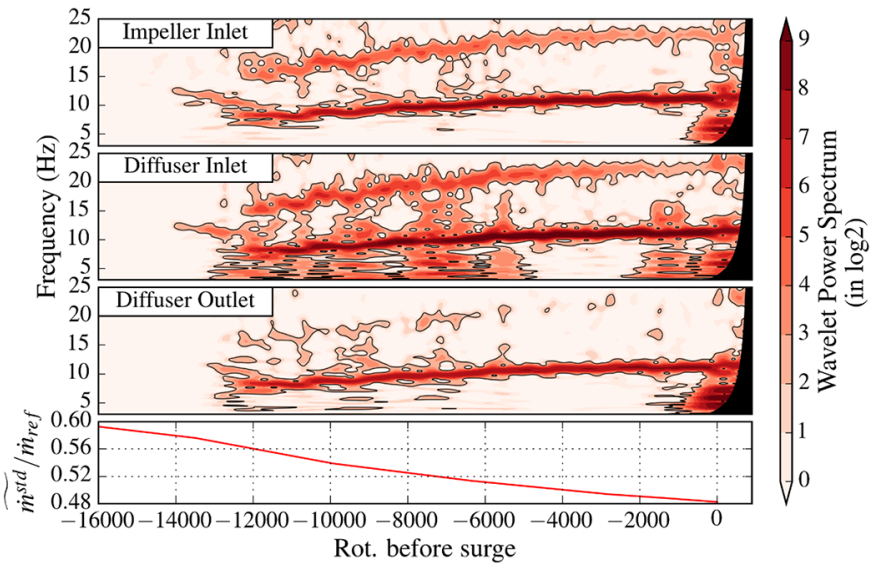

Fig. 4. Wavelet Power Spectrum during throttling ramp from stable condition to surge at isospeed C.

- For low alternate rate (white area; $\mathcal{A}<0.007$ ), a symmetric behaviour of the diffuser flow is observed (see Fig. 6). Every channel behaves like its neighbor and pressure fluctuations are measured on the whole compressor at a frequency of approximatively $42 \mathrm{~Hz}$.

- For high alternate rate (pink area; $\mathcal{A}>0.025$ ), the alternate pattern pulses inside the diffuser which operates successively in a periodic way and in an alternate way at a frequency of approximatively $12 \mathrm{~Hz}$ (see Fig. 9).

- For moderate alternate rate (blue area; $0.007<\mathcal{A}$ $<0.025$ ), complex mixed operation of the diffuser is observed with both $12 \mathrm{~Hz}$ and $42 \mathrm{~Hz}$ pressure fluctuations.

Those $12 \mathrm{~Hz}$ and $42 \mathrm{~Hz}$ modes are then described further in details in the following.

Figures 3 and 4 show the low-frequency wavelet power spectra calculated from the signals of sensors at the impeller inlet, diffuser inlet and diffuser outlet during a continuous throttling from a stable operating point up to surge at isospeed $\mathrm{A}$ and $\mathrm{C}$ respectively. At the bottom of each figure, the throttling ramp is given. Taking into
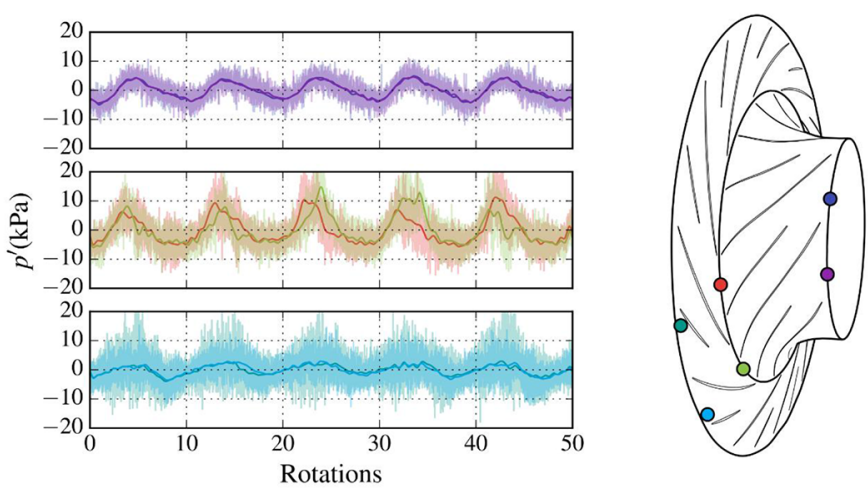

Fig. 5. Unsteady pressure fluctuations near surge during the spatially periodic mode at isospeed A.

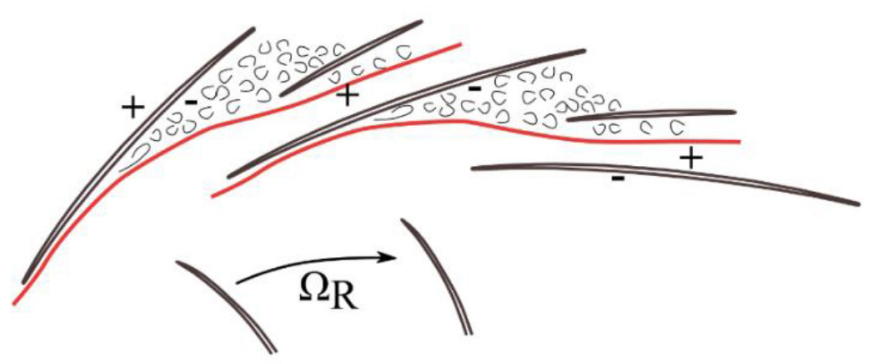

Fig. 6. Scheme of the flow topology in the radial diffuser during spatially periodic mode.

account the change in rotation speed, the valve ramps are substantially equivalent, leading to a $1 \%$ drop in flow rate in $2.5 \mathrm{~s}$. It is then obvious that the time of activity of instabilities is much longer at isospeed $\mathrm{C}$ than at isospeed A, with a factor of about 3 . This confirms what was visible in Figure 2, in that the mass flow range with instabilities is wider at isospeed $\mathrm{C}$ than at isospeed $\mathrm{A}$.

At isospeed C (Fig. 4), whether at the impeller inlet, diffuser inlet or diffuser outlet, the signals show a significant low frequency activity. First around $8 \mathrm{~Hz}$ (and harmonics), it evolves until it reaches $12 \mathrm{~Hz}$ (and harmonics) just before deep surge occurrence. Its intensity also increases with the reduction of the flow rate. Previous studies [15] linked this mode to the occurrence of mild surge inside the whole compressor. As described by Fink [16], it consists of longitudinal fluctuation of the mass flow without its complete reversal.

At isospeed A (Fig. 3), the spectra are first dominated by a frequency of about $42 \mathrm{~Hz}$. During the last thousand rotations before surge occurrence, multiple frequencies are superimposed on the previous one, first at the diffuser inlet before affecting the whole compressor. Finally, deep surge is registered with a frequency of about $8 \mathrm{~Hz}$.

\section{Analysis of the low frequency modes}

\subsection{Spatially periodic mode $(42 \mathrm{~Hz})$}

The $42 \mathrm{~Hz}$ mode is exclusively longitudinal without any rotation. Figure 5 gives the fluctuations of the 

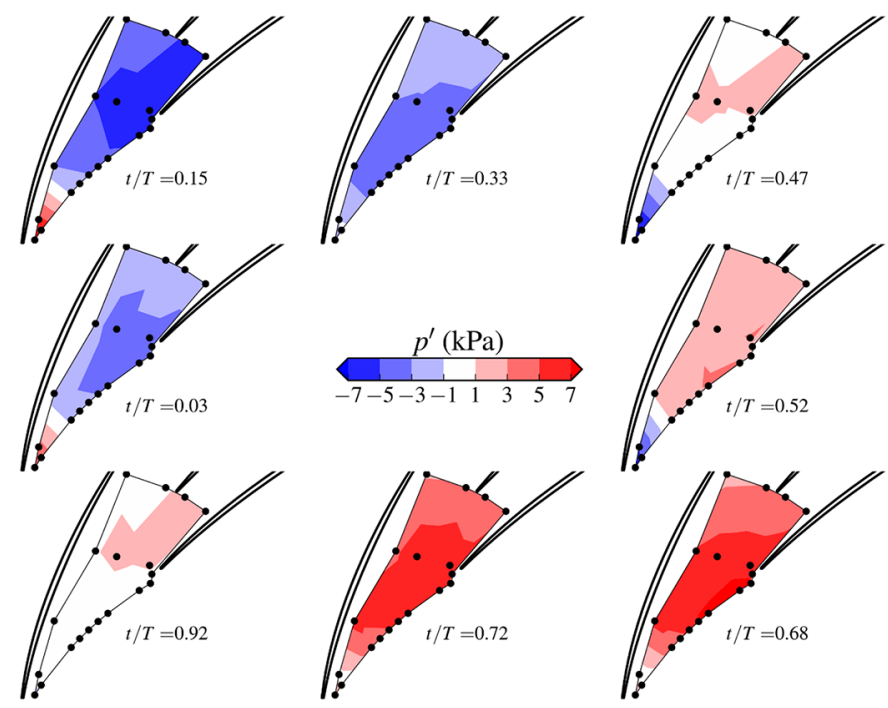

Fig. 7. Phase averaged unsteady pressure fluctuations in the diffuser at shroud during the spatially periodic mode at isospeed $\mathrm{A}$.

time-dependent pressure signals from two sensors at impeller inlet, two sensors at diffuser inlet and two sensors at diffuser outlet. At each section, no shift in phase is visible between the two signals, which reflects the lack of mode rotation. In addition, it should be noted that the amplitude of the strongest fluctuations is at the diffuser inlet.

Actually, a deeper analysis of the pressure signals delivered by the sensors in the diffuser, shows that the mode propagating throughout the machine with a frequency around $42 \mathrm{~Hz}$ results from the simultaneous oscillation of a separation occurring in every diffuser channel. To illustrate the situation, let us consider a sketch of a separation which could occur near the diffuser vane suction side, at a given time (Fig. 6). Each diffuser passage has the same flow structure.

During a period of the spatially periodic mode $(f \approx 42 \mathrm{~Hz}$ ), the separation line (red line in Fig. 6 ) oscillates in the vaned diffuser passage from a position very close the suction side up to be almost aligned with the vaned leading edges. This is highlighted by the phase-averaged fluctuations of the time-dependent pressure signals from the sensors located at shroud, during a period of the $42 \mathrm{~Hz}$ mode in Figure 7.

Pressure fluctuations during a period show that a major part of the diffuser passage simultaneously experiences fluctuations either positive or negative. The over-pressure phases (red areas) correspond to a reduction in flow velocity and therefore to an upstream migration of the separation. Whereas the under-pressure phases (blue area) correspond to an increase in velocity and therefore to a reduction of the separated flow area leading to a downstream migration of the separation line, close to the vane suction side.

\subsection{Alternate mode $(12 \mathrm{~Hz})$}

In a similar way to what was represented in Figure 5, Figure 8 gives the fluctuations of the time-dependent
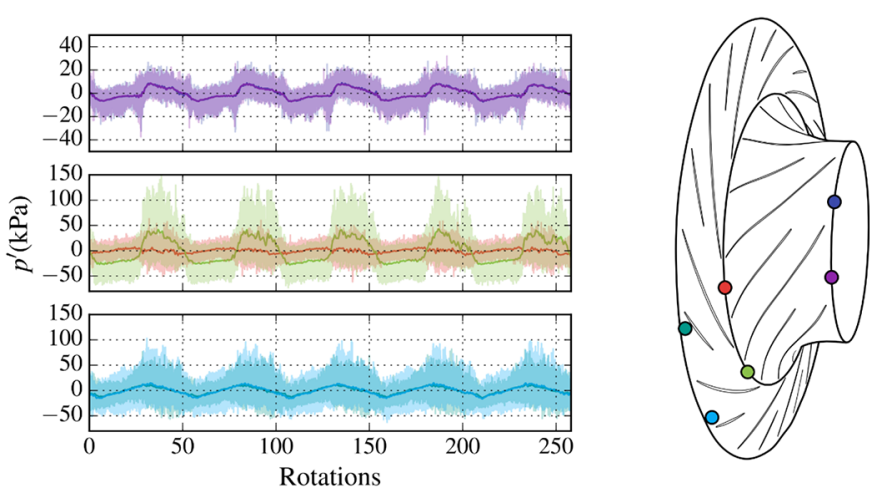

Fig. 8. Unsteady pressure fluctuation near surge during the alternate mode at isospeed $\mathrm{C}$.

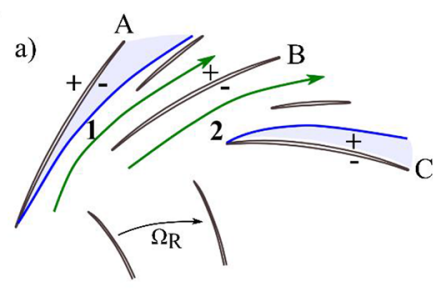

Quasi-periodic phase

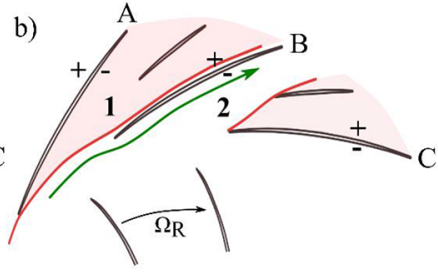

Alternate phase
Fig. 9. Scheme of the flow topology in the radial diffuser during alternate mode.

pressure signals at impeller inlet, diffuser inlet and diffuser outlet. Again, no shift in phase is visible which reflects the lack of mode rotation. By comparing Figures 5 and 8, it is clearly noted that the amplitude of the fluctuations is significantly higher in the $12 \mathrm{~Hz}$ mode case than in the $42 \mathrm{~Hz}$ mode case. A factor of more than 5 is measured upstream of the diffuser between the two cases. It must also be noted that the two probes at the diffuser inlet placed at different circumferential positions deliver very different signals. This reflects a much more complex flow structure than previously, as explained below.

Although the alternate $(f \approx 12 \mathrm{~Hz})$ mode is also a longitudinal mode without rotation, the associated flow structure is much more complex because the flow developing in the diffuser is periodic over two adjacent channels and no longer over one as previously or conventionally encountered [12]. In order to illustrate the situation, let us consider the separated flow structure shown in Figure 9.

A flow pattern over two adjacent channels develops in the diffuser and repeated around the entire circumference. It is characterized by:

- a stalled channel (channel 1) with a separation at its inlet inducing a low speed and a high pressure,

- a free-flow channel (channel 2) with a jet close to the suction side of blade B due to the deviation of the flow from channel 1 induced by the separation. The jet-flow is characterized by a low pressure close to the suction side of blade B.

During a period of the $12 \mathrm{~Hz}$ mode, the separation line in channel 1 oscillates from a position close the suction side of blade A (Fig. 9a) up to the channel 1 inlet (Fig. 9b). At that 

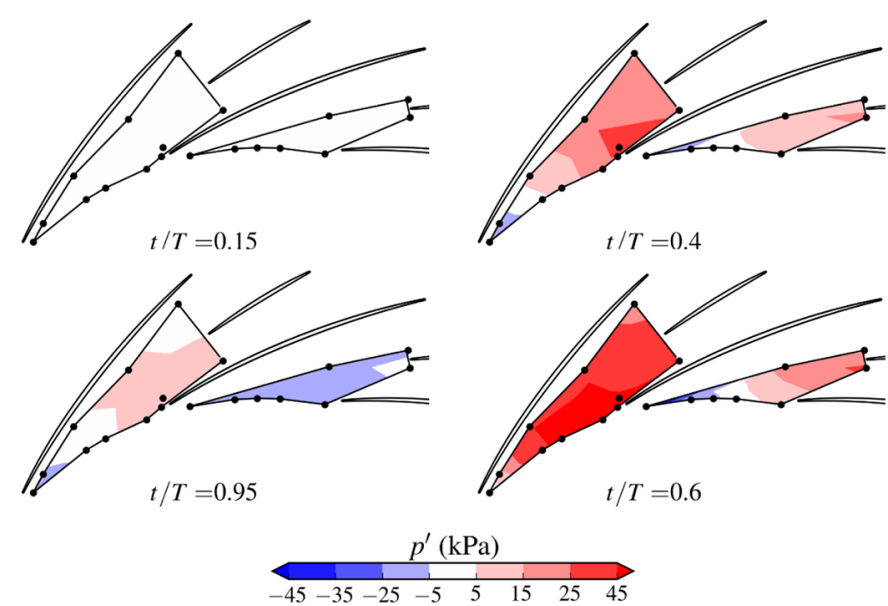

Fig. 10. Phase averaged unsteady pressure fluctuations in the diffuser at shroud during the alternate mode at isospeed $\mathrm{C}$.

time, the jet flow appears near the suction side of blade B as sketched in Figure 9b and the separation line in channel 2 moves towards the suction side of blade B. The blockage of channel 1 leads to a bad operation of the diffuser which pressure recovery drops. Since the pressure at the diffuser outlet is lower, the separated area is reduced and the separation line returns close to the suction side of channel 1. Then, as the diffuser works better, its outlet pressure increases, the separated area widens and the separation line moves upstream. This cycle is repeated at a frequency of $12 \mathrm{~Hz}$. The phase-averaged fluctuations of the timedependent pressure signals registered from the sensors located at shroud, during a period of the $12 \mathrm{~Hz}$ mode are given in Figure 10.

Due to the alternate flow pattern, two adjacent diffuser vane passages have to be considered. From a state without any fluctuation (at reduced time $t / T=0.15$ ), the alternate flow pattern is at its peak at $t / T=0.6$.

Actually both cases ( 12 and $42 \mathrm{~Hz}$ modes) correspond to a pulsating operation of the compressor due to the periodic fluctuation of the separation lines in the diffuser, the $42 \mathrm{~Hz}$ mode being associated with an spatially periodic flow pattern in the diffuser whereas the $12 \mathrm{~Hz}$ mode is associated with an alternate flow pattern. In both cases, as the mass flow is further reduced the surge occurs at a low frequency in the range of $6-8 \mathrm{~Hz}$. The following section aims at understanding the reason for the value of these frequencies of 12 and $42 \mathrm{~Hz}$.

\section{Acoustic lock-in}

The low frequency of the two modes led to an experimental characterization of the acoustic modes of the compressor test-rig.

\subsection{Experimental procedure}

As the engine is off, a detonation is generated at the inlet of the test-rig. Microphones of high sensitivity $(0.25 \mathrm{mV} / \mathrm{Pa})$ placed in the upstream pipe of the compressor then record the pressure fluctuations related to pressure waves
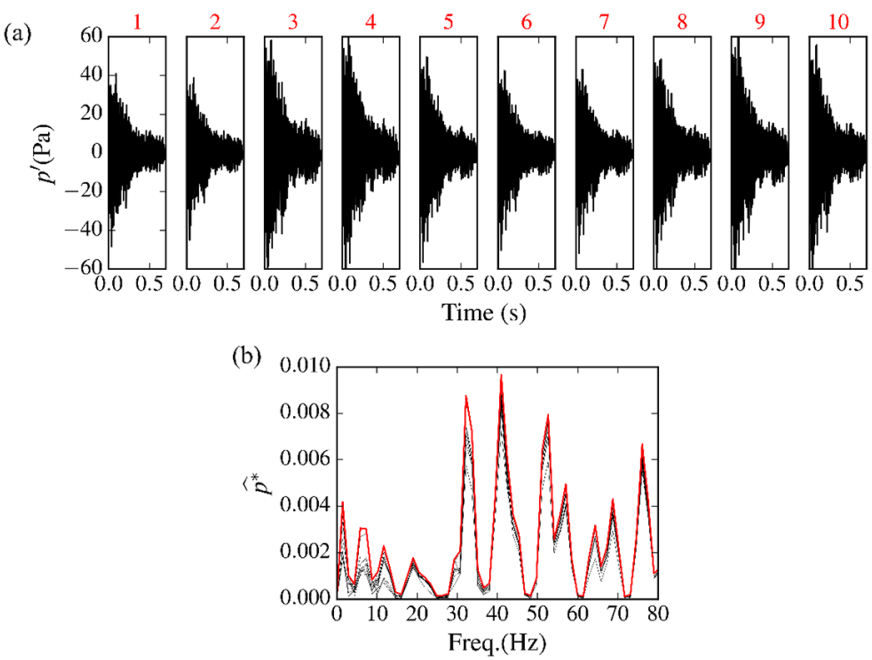

Fig. 11. Experimental characterization of the acoustic modes of the compressor test-rig.

propagations in the test rig. The detonation plays the role of a broadband exciter source and the test bench plays the role of resonator. Only the pressure waves admitted by the test bench acoustics are then propagated.

For post-processing, the useful part of the signal is recovered and normalized by the maximum value of the pressure fluctuations of the detonation. A Fourier transform is then performed on the normalized signal to determine the frequency of the acoustic waves propagating through the test rig. The operation is then repeated for 10 detonations.

\subsection{Results}

Figure 11a shows the useful parts of the signals from 10 detonations numerated in red for the sensor situated furthest upstream of the compressor. Figure 11b shows the Fourier transforms of all the normalized signals (defined in Eq. (3)) in black and the maximum of all Fourier transforms for each frequency in red

$$
p^{*}=\frac{p^{\prime}}{\left|p^{\prime}\right|_{\max }} .
$$

Many peaks of varying intensities are observed suggesting a complex acoustic behavior of the test rig. The most important one in the $0-80 \mathrm{~Hz}$ range corresponds to a frequency of $41 \mathrm{~Hz}$. This frequency is very close to the frequency of the oscillation of the symmetrical separation observed at isospeed A. A peak of smaller amplitude is also observed at $11.7 \mathrm{~Hz}$ corresponding to the frequency of the oscillation of the alternate stall at isospeed C. It has to be noted that these frequencies are found without flow in the bench. A slight difference will be induced by the influence of the average flow velocity in wave propagation. However, due to the disposition of the microphones, the flow rates in the compressor and the size of the pipe sections in the upstream part of the test rig, its influence can be negligible.

From these acoustic results, it is likely that the $42 \mathrm{~Hz}$ spatially periodic mode simply results from a coupling 
between the natural frequency of the instability and the test rig acoustics. Concerning the alternate mode, the natural frequency of the alternate stall is linked to a Helmholtz type resonance in the downstream circuit. Adopting the definitions of lengths and sections to be taken into account as defined in [16], the Helmholtz frequency is found to be around $16 \mathrm{~Hz}$. This lock-in between the alternate stall and the Helmhotz type resonance is suspected to maintain the pressure fluctuations throughout the test rig up to surge.

One way to confirm these assumptions could be to modify the geometry of the test bench sufficiently to change its acoustic modes and to verify whether a change in the frequency of instabilities is observed. If the downstream geometry is modified, it is certain that the frequency of the associated Helmholtz resonance will change, as shown by Galindo et al. [7]. Therefore, the most interesting thing would be to modify the upstream part of the test rig.

\section{Conclusion}

From high-frequency pressure measurements, a comprehensive description of the path to surge of a centrifugal compressor at partial rotation speeds has been proposed. Two specific but representative rotation speeds have been selected for the analysis. In one case, the vaned diffuser reveals an alternate flow pattern and the flow in the entire compressor pulses at $12 \mathrm{~Hz}$. In the second case, the vaned diffuser operates classically and the flow in the entire compressor pulses at $42 \mathrm{~Hz}$.

It is thought that the $42 \mathrm{~Hz}$ spatially periodic mode simply results from a coupling between the natural frequency of the instability and the test rig acoustics. The $12 \mathrm{~Hz}$ mode seems to result from a lock-in of the natural frequency of the alternate stall with the Helmhotz type resonance of the test rig.

These two cases show the importance of the system in which the compressor is included for the analysis of the surge: even if the origin of the perturbations triggering surge is indisputably due to the compressor (in the vaned diffuser in the present case), the surge development (i.e. the perturbations growth) is conditioned by the system.

An investigation of the impact of the IGV stagger angle on the onset and development of these modes will be carried out.

We would like to thank SAFRAN Helicopter Engines which supported this study.

\section{References}

[1] N.A. Cumpsty, Compressor aerodynamics, Longman Scientific \& Technical (1989)
[2] R. Hunziker, G. Gyarmathy, The operational stability of a centrifugal compressor and its dependence on the characteristics of the subcomponents, in International Gas Turbine and Aeroengine Congress and Exposition, American Society of Mechanical Engineers (1993)

[3] X. Zheng, A. Liu, Phenomenon and mechanism of tworegime-surge in a centrifugal compressor, J. Turbomach. 137 (2015)

[4] X. He, X. Zheng, Flow instability evolution in high pressure ratio centrifugal compressor with vaned diffuser, Exp. Thermal Fluid Sci. 98, 719-730 (2018)

[5] E.M. Greitzer, Surge and rotating stall in axial flow compressors-Part I: Theoretical compression system model, J. Eng Power 98, 190-198 (1976)

[6] E.M. Greitzer, Surge and rotating stall in axial flow compressors-Part II: experimental results and comparison with theory, J. Eng. Power 98, 199-211 (1976)

[7] J. Galindo, J.R. Serrano, H. Climent, A. Tiseira, Experiments and modelling of surge in small centrifugal compressor for automotive engines, Exp. Therm Fluid Sci. 32, 818-826 (2008)

[8] A. Godard, I. Trébinjac, M. Rouméas, Experimental characterization of the surge onset in a turbo-compressor for fuel cell application, in Proceedings of 12th European Conference on Turbomachinery, ETC, 2017, Stockholm, Sweden, 2017

[9] L. Zhang, J. Scott, G. Ngo Boum, Méthode de paramétrage du modèle Moore-Greitzer à partir d'un cycle de pompage fourni, in Congrès français de mécanique, Association Française de Mécanique, 2015

[10] S. Ziada, A. Oengören, A. Vogel, Acoustic resonance in the inlet scroll of a turbo-compressor, J. Fluids Struct. 16, 361-373 (2002)

[11] C. Brandstetter, B. Paoletti, X. Ottavy, Compressible modal instability onset in an aerodynamically mistuned transonic fan, J. Turbomach. 141 (2019)

[12] E. Benichou, I. Trébinjac, Numerical analysis of an alternate stall in a radial vaned diffuser, in Turbomachinery Technical Conference and Exposition. American Society of Mechanical Engineers (2016)

[13] V. Moënne-Loccoz, I. Trébinjac, E. Benichou, S. Goguey, B. Paoletti, P. Laucher, An experimental description of the flow in a centrifugal compressor from alternate stall to surge, J. Therm. Sci. 26, 289-296 (2017)

[14] V. Moënne-Loccoz, I. Trébinjac, P. Laucher, Experimental application of a boundary layer control strategy in a radial vaned diffuser, in Turbomachinery Technical Conference and Exposition, American Society of Mechanical Engineers (2018)

[15] V. Moënne-Loccoz, I. Trébinjac, N. Poujol, P. Duquesne, Detection and analysis of an alternate flow pattern in a radial vaned diffuser, Int. J. Turbomach. Prop. Power 5, 2 (2020)

[16] D.A. Fink, N.A. Cumpsty, E.M. Greitzer, Surge dynamics in a free-spool centrifugal compressor system, in: International Gas Turbine and Aeroengine Congress and Exposition, American Society of Mechanical Engineers (1991)

Cite this article as: V. Moënne-Loccoz, I. Trébinjac, N. Poujol, P. Duquesne, Low frequency stall modes of a radial vaned diffuser flow, Mechanics \& Industry 20, 805 (2019) 